\title{
Transtorno mental comum em mulheres adultas: identificando os segmentos mais vulneráveis
}

\author{
Common mental disorders in adult women: \\ identifying the most vulnerable segments
}

Caroline Senicato ${ }^{1}$

Renata Cruz Soares de Azevedo ${ }^{2}$

Marilisa Berti de Azevedo Barros ${ }^{1}$

${ }^{1}$ Departamento de Saúde Coletiva, Faculdade de Ciências Médicas, Unicamp. R. Tessália Vieira de Camargo, Cidade Universitária. 13083-887 Campinas SP Brasil.carolinesenicato@ hotmail.com

${ }^{2}$ Departamento de

Psicologia Médica e

Psiquiatria, Faculdade de

Ciências Médicas, Unicamp.

Campinas SP Brasil.

\begin{abstract}
This study aimed to evaluate the socioeconomic and demographic factors, behaviors and morbidities related to common mental disorders in adult women. This was a cross-sectional population-based study with cluster sample. We analyzed 848 women from a household survey held in Campinas, in 2008/2009. We used the Self-Reporting Questionnaire (SRQ-20) to evaluate common mental disorders. We estimated prevalence ratios by Poisson regression in hierarchical model of three steps, considering the weights relating to the sampling design. The prevalence of common mental disorders was $18.7 \%$. The hierarchical model showed that older women, with low education level, housewives, separated or widowed, who did not consume fruit/vegetables daily, who slept six or fewer hours per night, who presented several chronic diseases and health problems, and with report of some type of violence were more vulnerable to common mental disorders and, therefore, should be treated with priority by health services. Early diagnosing women with common mental disorders, as well as accompanying and treating them, contribute for reducing the impacts on female quality of life.
\end{abstract}

Key words Women's health, Mental disorders, Mental health, Prevalence, Health surveys
Resumo O objetivo deste estudo foi avaliar os fatores socioeconômicos e demográficos, os comportamentos e as morbidades associados ao transtorno mental comum em mulheres adultas. Trata-se de um estudo transversal de base populacional com amostra por conglomerados. Foram analisadas 848 mulheres por inquérito domiciliar realizado em Campinas, em 2008/2009. Foi usado o instrumento Self-Reporting Questionnare (SRQ-20) para avaliar o transtorno mental comum. Foram estimadas razões de prevalências por meio de regressão múltipla de Poisson em modelo hierárquico de três etapas, considerando as ponderaçôes relativas ao desenho amostral. A prevalência de transtorno mental comum foi de $18,7 \%$. O modelo hierárquico evidenciou que mulheres mais velhas, com baixa escolaridade, donas de casa, separadas ou viúvas, que não consumiam frutas/ verduras/legumes diariamente, dormiam seis ou menos horas por noite, apresentavam várias doenças crônicas e problemas de saúde e com relato de algum tipo de violência foram mais vulneráveis ao transtorno mental comum e, por isso, devem ser tratadas com prioridade pelos serviços de saúde. Diagnosticar precocemente mulheres com transtorno mental comum, bem como acompanhá-las e tratá-las, contribuem para reduzir os impactos na qualidade de vida feminina.

Palavras-chave Saúde da mulher, Transtornos mentais, Saúde mental, Prevalência, Inquéritos epidemiológicos 


\section{Introdução}

Segundo a Organização Mundial da Saúde (OMS) uma em cada quatro pessoas desenvolverá algum transtorno mental durante a vida ${ }^{1}$. Caracterizados por sintomas depressivos, estado de ansiedade e um conjunto de queixas somáticas inespecíficas ${ }^{2}$, o transtorno mental comum (TMC) é mais elevado nas mulheres do que nos homens ${ }^{3}$. Os transtornos mentais são a principal fonte de anos de vida saudáveis perdidos (DALY) para as mulheres entre 15 e 24 anos $^{4}$. Prevê-se que, até 2020, a depressão passe a ser a primeira causa de DALY em mulheres em idade reprodutiva de países em desenvolvimento, na frente dos problemas de saúde diretamente relacionados à gestação, parto e puerpério ${ }^{5}$. Os prejuízos dos transtornos mentais na qualidade de vida decorrem do comprometimento funcional com perda de produtividade no trabalho e isolamento social, e conduzem ao aumento da utilização de serviços de saúde ${ }^{6,7}$, que produz custos elevados para o sistema de saúde e para os indivíduos e suas famílias, e custos menos mensuráveis, como o sofrimento individual e do grupo familiar.

De acordo com uma meta-análise que avaliou 174 publicações, de 1980 a 2013, de 63 países de alta, média e baixa renda, 17,6\% dos adultos apresentaram transtornos mentais comuns nos últimos 12 meses, entre as mulheres essa prevalência foi de 19,7\%. Os autores destacaram que, independente da situação econômica do país, as mulheres apresentaram maior prevalência de transtornos de humor e de ansiedade do que os homens ${ }^{3}$.

A maior vulnerabilidade feminina aos transtornos mentais pode ser devido às alterações no sistema endócrino que ocorrem no período prémenstrual, pós-parto e menopausa; às pequenas diferenças no cérebro, com algumas características mais comuns no de mulheres do que de homens $^{8}$; e, às desigualdades de gênero, que têm dentre as consequências desde a sobrecarga de trabalho doméstico ${ }^{9}$ às altas taxas de violência ${ }^{10}$. Além dos fatores biológicos e sociais que podem colaborar para a diferença entre os percentuais de transtornos mentais entre homens e mulheres, alguns fatores de risco parecem ser comuns para ambos, como as condições e suporte psicossociais, situação socioeconômica, estilo de vida e a situação de saúde ${ }^{11}$.

Apesar de se compreender que a mulher apresenta maior vulnerabilidade, é preciso melhor entendimento das condições socioeconômicas, estilo de vida e comorbidades associadas aos transtornos mentais, particularmente entre elas. Poucos estudos brasileiros investigaram o transtorno mental comum especificamente na população feminina, de acordo com uma revisão sistemática de 1997 a 2009 sobre transtornos mentais comuns na população adulta brasileira, os autores identificaram somente três estudos específicos com mulheres, todos realizados no nordeste do Brasil, que avaliaram a prevalência de TMC segundo inserção no mercado de trabalho, com resultados superiores aos mundiais, variando entre 28,1 e $36,8 \%$ no último ano ${ }^{12}$.

Embora existam domínios específicos dos transtornos mentais, como depressão e transtorno de ansiedade, este artigo centra-se nos TMC rastreados pelo instrumento Self-Reporting Questionnare (SRQ-20), amplamente utilizado em inquéritos de saúde de base populacional. Objetivou-se avaliar a prevalência de TMC entre as mulheres do município de Campinas e identificar a associação desse transtorno com variáveis socioeconômicas e demográficas, comportamentos relacionados à saúde, estado de saúde e morbidades. Conhecer os segmentos de mulheres mais vulneráveis ao transtorno mental comum permite o planejamento de ações voltadas à saúde da mulher que minimizem o prejuízo para a qualidade de vida em todo o ciclo de vida feminino, bem como os gastos do sistema público de saúde.

\section{Métodos}

Trata-se de um estudo transversal de base populacional, que utilizou dados do Inquérito de Saúde do Município de Campinas (ISACamp), realizado entre 2008 e 2009.

O processo de amostragem do ISACamp 2008/2009 foi em dois estágios. No primeiro estágio, foram sorteados 50 setores censitários da área urbana do município de Campinas com probabilidade proporcional ao número de domicílios. Foi feito um sorteio sistemático, no qual os setores foram ordenados pelo percentual de chefes de família com nível universitário. No segundo estágio da amostra, após elaboração de listagem atualizada dos endereços dos domicílios dos setores censitários selecionados, foram sorteados os domicílios.

O tamanho da amostra foi definido considerando a estimativa de uma proporção de 0,50 , com um erro máximo de 4 a 5 pontos percentuais, com intervalo de confiança de $95 \%$ e efeito de delineamento de 2 , resultando em 1.000 indi- 
víduos para cada domínio de idade: adolescente (10 a 19 anos), adulto (20 a 59 anos) e idoso (60 anos ou mais). Esperando $80 \%$ de taxa de resposta, o tamanho da amostra foi corrigido para 2.150, 700 e 3.900 domicílios, respectivamente para entrevistas com adolescentes, adultos e idosos. Foram entrevistados todos os residentes do domicílio, que fossem do domínio de idade sorteado para aquele domicílio (maiores detalhes do plano amostral encontram-se em: http:// www.fcm.unicamp.br/fcm/sites/default/files/ plano_de_amostragem.pdf). No presente estudo foram utilizados somente os dados de adultos do sexo feminino na faixa de 18 a 64 anos de idade, totalizando 848 mulheres na amostra.

Informações demográficas, socioeconômicas, de comportamentos relacionados à saúde, de condições de saúde e de uso de serviços de saúde foram obtidas por meio de um questionário estruturado, previamente testado, aplicado por entrevistadores treinados e supervisionados. A entrevista foi feita diretamente com a mulher selecionada.

As variáveis analisadas neste estudo foram:

- Transtorno mental comum: a presença de TMC foi avaliada utilizando-se o instrumento Self Reporting Questionnaire 20 (SRQ-20) desenvolvido pela Organização Mundial da Saúde para o rastreamento dos casos suspeitos de TMC em países em desenvolvimento. As questões foram referentes aos últimos trinta dias. $\mathrm{O}$ instrumento SRQ-20 é composto por 20 questões. As respostas são dicotômicas e para cada resposta afirmativa é atribuído um ponto, totalizando-se 20 pontos no caso de todas as respostas serem positivas. Foram considerados possíveis casos de TMC as mulheres que pontuaram seis ou mais pontos ${ }^{13}$.

- Variáveis demográficas e socioeconômicas: faixa etária ( 18 a 29; 30 a 49 e 50 a 64), cor da pele/ raça autorreferida (branca, preta e parda. Foram excluídas as de cor da pele/raça amarela devido ao pequeno número de mulheres, $\mathrm{n}=4$ ), religião (católica, evangélica, sem religião e outras), escolaridade (em anos de estudo e categorizada em: 0 a 8, 9 a 12 e 13 ou mais anos), ocupação (trabalhadoras remuneradas, desempregadas, aposentadas, donas de casa e estudantes. Foram classificadas como trabalhadoras remuneradas as mulheres que declararam estar exercendo uma atividade remunerada, mesmo que no momento da pesquisa estivessem temporariamente afastadas por motivo de doença ou aposentadas, mas que continuavam trabalhando de forma remunerada; como desempregadas, as mulheres que não estavam trabalhando, mas declararam estar procurando emprego; aposentadas, as mulheres que não trabalhavam mais de forma remunerada e estavam recebendo o salário de aposentadoria da previdência social ou da previdência privada; donas de casa, as mulheres que não exerciam nenhuma atividade remunerada e declararam ser donas de casa; e, estudantes, aquelas que não exerciam nenhuma atividade remunerada e só se dedicavam aos estudos), renda familiar per capita (calculada em número de salários mínimos (SM) e categorizada em: $\geq 2$ SM e $<2$ SM), estado conjugal (casada, vive junto/amasiada, desquitada/ separada/divorciada/viúva e solteira), número de filhos (nenhum, 1 a 2 e 3 ou mais), número de moradores do domicílio (1 a 2, 3 a 4 e 5 ou mais), presença de empregada doméstica (sim e não) e número de equipamentos no domicílio (11 ou mais, 6 a 10 e 5 a 0$)$.

- Comportamentos relacionados à saúde: consumo de risco de álcool avaliado pelo Alcohol Use Disorder Identification Test (AUDIT), composto por dez itens, considerado positivo com 8 pontos ou mais ${ }^{14}$; frequência de consumo de bebida alcoólica (não bebe, $\leq 1 \mathrm{vez} / \mathrm{semana}$ e $\geq 2$ vezes/semana); hábito de fumar (nunca fumou, fumante atual e ex-fumante), considerou-se não fumantes aquelas que referiram nunca ter fumado, ex-fumantes as que fumaram mais de 100 cigarros durante a vida, mas haviam cessado o consumo e, fumantes, as que informaram o uso atual de cigarro; prática de atividade física no contexto de lazer (ativa/insuficientemente ativa e inativa), avaliada pelo International Physical Activity Questionnaire (IPAQ), traduzido e submetido a estudo de validação no Brasil ${ }^{15}$, segundo a $\mathrm{OMS}^{16}$, foram consideradas mulheres ativas as que praticavam ao menos 150 minutos de atividade física moderada ou 75 minutos de atividade física vigorosa por semana, em sessões de pelo menos 10 minutos de duração, insuficientemente ativas: as que realizavam atividade física abaixo desse nível, e, inativas, as que não praticavam atividade física de lazer; consumo de frutas, verduras ou legumes (diário e não diário), segundo Ministério da Saú$\mathrm{de}^{17}$; e, duração média diária do sono $(\geq 7$ horas $\mathrm{e} \leq 6$ horas).

- Estado de saúde e morbidades: doenças crônicas (não e sim), referidas como tendo sido diagnosticadas por médico ou outro profissional de saúde, listadas em um checklist: hipertensão arterial, diabetes, doença do coração, reumatismo/artrite/artrose, asma/bronquite/enfisema, tendinite/lesão por esforço repetitivo (LER)/ distúrbio osteomuscular relacionado ao trabalho (DORT) e problema circulatório; e, número de 
doenças crônicas referidas entre as relacionadas (nenhuma, 1, 2, 3 e 4 ou mais); problemas de saúde referidos (não e sim): dor de cabeça/enxaqueca, dor nas costas/problema de coluna, alergia, tontura/vertigem, insônia e problema urinário; e, número de problemas de saúde autorreferidos entre os relacionados ( 2 ou menos, 3,4 e 5 ou mais); ocorrência de acidente e de violência no último ano (não e sim); índice de massa corporal (IMC), calculado com as informações autorreferidas de peso e altura (eutrófico: IMC $\geq 18,5$ a $<25 \mathrm{~kg} / \mathrm{m}^{2}$; baixo peso: IMC $<18,5 \mathrm{~kg} / \mathrm{m}^{2}$; sobrepeso: IMC $\geq 25 \mathrm{a}<30 \mathrm{Kg} / \mathrm{m}^{2}$; e obeso: IMC $\geq 30 \mathrm{~kg} / \mathrm{m}^{2}$ ); e, autoavaliação da saúde (excelente, muito boa, boa e ruim/muito ruim).

Neste estudo, a variável dependente foi transtorno mental comum, avaliado pelo SRQ-20. As variáveis independentes foram os indicadores demográficos e socioeconômicos, os comportamentos e as condições de saúde e morbidades; a idade (contínua) foi usada para controle de confundimento nas análises bivariadas e mantida no modelo final.

As associações entre as variáveis foram determinadas por meio do teste qui-quadrado, com nível de significância de 5\%. Modelos de regressão múltipla de Poisson com variância robusta foram usados para estimar as razões de prevalências ajustadas e seus intervalos de confiança de $95 \%$. Foi realizado um modelo hierárquico de regressão de Poisson em três etapas. Na primeira etapa do modelo foram introduzidas as variáveis socioeconômicas e demográficas com $\mathrm{p}<0,20$ na análise bivariada; aquelas que apresentaram $\mathrm{p}<$ 0,05 na associação com o TMC permaneceram no modelo. Na segunda etapa, além das variáveis que permaneceram na etapa anterior, acrescentaram-se os comportamentos relacionados à saúde com $\mathrm{p}<0,20$ e mantiveram-se aqueles com $\mathrm{p}<$ 0,05 . Na terceira etapa foram incluídos o número de doenças crônicas, o número de problemas de saúde referidos, IMC, e acidente e violência sofridos no último ano mantendo-se no modelo as variáveis que apresentaram um nível de significância menor de $5 \%(\mathrm{p}<0,05)$. As análises estatísticas, realizadas com o programa Stata versão 11.0 (Stata Corp., College Station, Estados Unidos), módulo svy, consideraram os pesos amostrais e o efeito do delineamento do estudo. Esta pesquisa foi aprovada pelo Comitê de Ética da Faculdade de Ciências Médicas da Universidade Estadual de Campinas. O Termo de Consentimento Livre e Esclarecido foi assinado por todas as participantes da pesquisa.

\section{Resultados}

A amostra deste estudo foi composta por 848 mulheres com idades entre 18 e 64 anos. A prevalência de TMC entre as mulheres adultas no município de Campinas, avaliada pelo SRQ-20, foi de 18,7\% (IC95\%: 14,2-23,2).

Na Tabela 1, após ajuste por idade, as razões de prevalências de TMC foram significativamente maiores em mulheres com trinta anos ou mais, que referiram raça/cor preta, com até doze anos de escolaridade, que eram donas de casa, com renda familiar per capita menor que dois salários mínimos, desquitadas/separadas/divorciadas/viúvas, e que tinham três ou mais filhos, comparadas às respectivas categorias de referência.

Dentre os comportamentos relacionados à saúde, as mulheres que eram fumantes, inativas fisicamente no lazer, que não consumiam frutas, verduras ou legumes diariamente e que dormiam seis horas ou menos por noite apresentaram razões de prevalências de TMC significativamente maior, após o ajuste por idade (Tabela 2).

A Tabela 3 apresenta a prevalência de TMC segundo doenças crônicas, problemas de saúde referidos, acidentes e violência, índice de massa corporal e autoavaliação da saúde. Após o ajuste por idade, as mulheres com diagnóstico médico de hipertensão, doença do coração, reumatismo/ artrite/artrose, asma/bronquite/enfisema, tendinite/LER/DORT e problema circulatório, e que relataram dor de cabeça, dor nas costas, tontura/vertigem, insônia e problema urinário apresentaram maior prevalência de TMC. Dentre as morbidades crônicas, as maiores prevalências de transtornos mentais foram verificadas em mulheres com problema circulatório $(\mathrm{RP}=2,58)$, tendinite/LER/DORT $(\mathrm{RP}=2,52)$ e reumatismo/artrite/artrose $(\mathrm{RP}=2,48)$. Já em relação aos problemas de saúde referidos, as mulheres com insônia se destacaram por apresentarem 4,07 vezes mais prevalência de TMC comparada àquelas que não referiram esse problema, seguidas pelas mulheres com problema urinário $(\mathrm{RP}=2,79) \mathrm{e}$ por aquelas que relataram tontura/vertigem (RP $=2,69$ ). Observou-se aumento importante da prevalência de TMC com o aumento do número de doenças crônicas e do número de problemas de saúde. Possuir quatro doenças crônicas ou mais aumentou 7,69 vezes a ocorrência de TMC e referir cinco ou mais problemas de saúde representou 2,86 vezes mais transtorno mental. As mulheres que sofreram violência no ano anterior à pesquisa apresentaram prevalência significativamente maior de TMC $(\mathrm{RP}=2,82)$, em relação 
Tabela 1. Prevalência e razão de prevalência (RP) de transtorno mental comum (SRQ-20) segundo variáveis socioeconômicas e demográficas de mulheres com 18 a 64 anos de Campinas, São Paulo. ISACamp 2008/2009.

\begin{tabular}{|c|c|c|c|}
\hline Variáveis & $\mathbf{n}^{*}$ & Prevalência (\%) & RP ajustada ${ }^{a}$ (IC 95\%) \\
\hline Faixa etária (anos) & & $0,0055^{\star \star}$ & \\
\hline 18 a 29 & 234 & 11,8 & 1 \\
\hline 30 a 49 & 257 & 20,0 & $1,69(1,04-2,74)$ \\
\hline 50 a 64 & 357 & 25,4 & $2,15(1,40-3,31)$ \\
\hline Total & 848 & 18,7 & \\
\hline Cor da pele/raça & & $0,1444^{* *}$ & \\
\hline Branca & 617 & 17,0 & 1 \\
\hline Preta & 66 & 25,9 & $1,54(1,01-2,34)$ \\
\hline Parda & 159 & 22,7 & $1,40(0,89-2,21)$ \\
\hline Religião & & $0,3936^{\star *}$ & \\
\hline Católica & 453 & 16,1 & 1 \\
\hline Evangélica & 278 & 21,5 & $1,40(0,96-2,03)$ \\
\hline Sem religião & 64 & 23,6 & $1,61(0,84-3,08)$ \\
\hline Outras & 50 & 18,1 & $1,17(0,54-2,50)$ \\
\hline Escolaridade (anos) & & $<0,0001^{\star *}$ & \\
\hline 13 ou mais & 200 & 7,7 & 1 \\
\hline 9 a 12 & 220 & 19,6 & $2,68(1,70-4,23)$ \\
\hline 0 a 8 & 427 & 25,5 & $2,99(1,77-5,04)$ \\
\hline Ocupação & & $0,0024^{\star \star}$ & \\
\hline Trabalhadoras remuneradas & 423 & 14,3 & 1 \\
\hline Desempregadas & 38 & 17,6 & $1,34(0,55-3,26)$ \\
\hline Aposentadas & 101 & 32,7 & $1,82(0,96-3,44)$ \\
\hline Donas de casa & 245 & 27,2 & $1,79(1,16-2,75)$ \\
\hline Estudantes & 34 & 11,2 & $0,98(0,37-2,61)$ \\
\hline Renda familiar per capita (SM) & & $0,0178^{\star \star}$ & \\
\hline$>=2$ & 259 & 13,0 & 1 \\
\hline$<2$ & 589 & 21,4 & $1,68(1,11-2,53)$ \\
\hline Estado conjugal & & $0,0067^{\star *}$ & \\
\hline Casada & 377 & 17,4 & 1 \\
\hline Vive junto/amasiada & 103 & 18,4 & $1,20(0,74-1,94)$ \\
\hline Desquitada/separada/divorciada/viúva & 146 & 33,0 & $1,75(1,12-2,74)$ \\
\hline Solteira & 222 & 13,8 & $0,96(0,52-1,76)$ \\
\hline Número de filhos & & $<0,0001^{\star *}$ & \\
\hline Nenhum & 224 & 10,2 & 1 \\
\hline 1 a 2 & 366 & 18,2 & $1,68(1,00-2,81)$ \\
\hline 3 ou mais & 258 & 28,9 & $2,51(1,38-4,56)$ \\
\hline No de moradores no domicílio (pessoas) & & $0,7123^{* *}$ & \\
\hline 1 a 2 & 263 & 19,8 & $1,05(0,68-1,64)$ \\
\hline 3 a 4 & 368 & 17,3 & 1 \\
\hline 5 ou mais & 217 & 20,2 & $1,22(0,84-1,77)$ \\
\hline Presença de empregada doméstica & & $0,1040^{* *}$ & \\
\hline $\operatorname{Sim}$ & 80 & 10,9 & 1 \\
\hline Não & 768 & 19,5 & $1,93(0,91-4,09)$ \\
\hline Número de equipamentos no domicílio & & $0,4345^{\star *}$ & \\
\hline 11 ou mais & 145 & 17,0 & 1 \\
\hline 6 a 10 & 457 & 20,9 & $1,32(0,84-2,06)$ \\
\hline 5 a 0 & 245 & 16,1 & $1,06(0,58-1,94)$ \\
\hline
\end{tabular}

${ }^{\star}$ Número de indivíduos na amostra não ponderada; ${ }^{* *}$ Teste qui-quadrado: valor de p. ${ }^{a}$ Modelo de regressão múltipla de Poisson. Variável de ajuste: idade. Salário mínimo vigente: janeiro a abril/2008: R\$415,00; maio/2008 a abril/2009: R\$ 450,00. 
Tabela 2. Prevalência e razão de prevalência (RP) de transtorno mental comum (SRQ-20) segundo comportamentos relacionados à saúde de mulheres com 18 a 64 anos de Campinas, São Paulo. ISACamp 2008/2009.

\begin{tabular}{|c|c|c|c|}
\hline Variáveis & $\mathbf{n}^{*}$ & Prevalência (\%) & RP ajustada ${ }^{a}$ (IC 95\%) \\
\hline Consumo de risco de álcool (AUDIT) & & $0,2503^{\star \star}$ & \\
\hline Não & 822 & 18,3 & 1 \\
\hline Sim & 24 & 30,5 & $1,79(0,78-4,11)$ \\
\hline Frequência de consumo de álcool & & $0,7064^{\star *}$ & \\
\hline Não bebe & 688 & 19,4 & 1 \\
\hline $1 \mathrm{vez} / \mathrm{sem}$ ou menos & 123 & 16,3 & $0,87(0,54-1,42)$ \\
\hline 2 ou mais vezes/sem & 35 & 16,2 & $0,81(0,34-1,93)$ \\
\hline Hábito de fumar & & $0,0147^{\star \star}$ & \\
\hline Nunca fumou & 633 & 15,6 & 1 \\
\hline Fumante & 129 & 27,9 & $1,70(1,17-2,47)$ \\
\hline Ex-fumante & 85 & 25,0 & $1,51(0,88-2,57)$ \\
\hline Atividade física de lazer (IPAQ) & & $0,0424^{\star *}$ & \\
\hline Ativa/Insuficientemente ativa & 244 & 12,1 & 1 \\
\hline Inativa & 604 & 21,0 & $1,86(1,08-3,21)$ \\
\hline Consumo de fruta/verdura/legumes & & $0,0262^{\star *}$ & \\
\hline Diário & 226 & 11,3 & 1 \\
\hline Não diário & 622 & 21,1 & $2,18(1,24-3,85)$ \\
\hline Duração diária do sono (horas) & & $<0,0001^{\star *}$ & \\
\hline$\geq 7$ & 678 & 14,7 & 1 \\
\hline$\leq 6$ & 165 & 34,2 & $2,20(1,51-3,21)$ \\
\hline
\end{tabular}

${ }^{\star}$ Número de indivíduos na amostra não ponderada; ${ }^{*}$ Teste qui-quadrado: valor de p. ${ }^{a}$ Modelo de regressão múltipla de Poisson. Variável de ajuste: idade.

à ausência dessa situação. As mulheres obesas apresentaram 1,67 vezes mais TMC do que as mulheres eutróficas. Já àquelas que avaliaram a própria saúde como boa ou ruim/muito ruim apresentaram respectivamente, 2,80 vezes e 10,16 vezes mais prevalência de transtorno mental comum, em comparação ao segmento de mulheres adultas que consideravam sua saúde excelente.

No modelo hierárquico de regressão múltipla de Poisson verificou-se maior prevalência de TMC nas mulheres de 30 a 49 anos, com até doze anos de escolaridade, que eram donas de casa, separadas/divorciadas/viúvas, que não consumiram frutas, verduras ou legumes diariamente, que dormiam seis horas ou menos por noite, que apresentaram uma ou mais doença crônica, quatro ou mais problemas de saúde referidos, e sofreram algum tipo de violência física ou psicológica no último ano que antecedeu a entrevista (Tabela 4).

\section{Discussão}

A prevalência de transtorno mental comum nas mulheres adultas no município de Campinas es- teve dentro da faixa encontrada por uma revisão sistemática sobre a prevalência dos transtornos mentais na população feminina brasileira, que foi de $19 \%$ a $34 \%^{12}$, e muito próxima ao valor encontrado por uma meta-análise, que avaliou pessoas de 16 a 65 anos em todo o mundo e concluiu que $19,7 \%$ das mulheres apresentaram algum transtorno mental comum no último ano ${ }^{3}$.

Como a literatura aponta, são muitos os fatores sociais envolvidos na saúde mental e no bem-estar das mulheres ao longo da vida ${ }^{18,19}$. Contudo, este estudo concluiu que, no conjunto dos determinantes sociais da saúde, alguns apresentam maior relevância do que outros para a saúde mental feminina. Analisando os indicadores socioeconômicos e demográficos relacionados aos TMC, observou-se que idade, cor da pele/raça, nível de escolaridade, ocupação, renda familiar per capita, estado conjugal e número de filhos estavam estatisticamente associados a esses transtornos. Entretanto, somente escolaridade e ocupação permaneceram significativas ao ajustar por todas as variáveis. As mulheres com até 8 anos de estudos apresentaram prevalência de TMC 2,67 vezes mais elevada do que aquelas com 13 anos ou mais. Mas, o que faz com que a 
Tabela 3. Prevalência e razão de prevalência (RP) de transtorno mental comum (SRQ-20)segundo morbidades e estado de saúde de mulheres com 18 a 64 anos de Campinas, São Paulo. ISACamp 2008/2009.

\begin{tabular}{|c|c|c|c|c|}
\hline Variáveis & $\mathbf{n}^{*}$ & Prevalência de TMC (\%) & $\mathbf{p}^{\star *}$ & RP ajustada $($ IC 95\%) \\
\hline Hipertensão arterial & 213 & 30,7 & 0,0016 & $1,62(1,07-2,47)$ \\
\hline Diabetes & 69 & 32,9 & 0,0090 & $1,48(0,98-2,25)$ \\
\hline Doença do coração & 52 & 38,4 & 0,0019 & $1,85(1,20-2,86)$ \\
\hline Reumatismo/artrite/artrose & 94 & 48,8 & $<0,0001$ & $2,48(1,59-3,88)$ \\
\hline Asma/bronquite/enfisema & 37 & 41,6 & 0,0328 & $2,16(1,05-4,41)$ \\
\hline Tendinite/LER/DORT & 62 & 45,3 & $<0,0001$ & $2,52(1,70-3,72)$ \\
\hline Problema circulatório & 138 & 43,1 & $<0,0001$ & $2,58(1,76-3,78)$ \\
\hline Número de doenças crônicas & & & $<0,0001$ & \\
\hline Nenhuma & 405 & 9,5 & & 1 \\
\hline 1 & 214 & 23,7 & & $2,58(1,66-3,99)$ \\
\hline 2 & 110 & 31,1 & & $3,52(1,90-6,49)$ \\
\hline 3 & 66 & 36,6 & & $4,19(2,56-6,88)$ \\
\hline 4 ou mais & 53 & 65,5 & & $7,69(4,53-13,06)$ \\
\hline Dor de cabeça/enxaqueca & 279 & 29,4 & $<0,0001$ & $2,29(1,77-2,97)$ \\
\hline Dor nas costas/problema de coluna & 308 & 27,2 & $<0,0001$ & $1,76(1,29-2,41)$ \\
\hline Alergia & 259 & 23,0 & 0,0844 & $1,40(0,98-2,00)$ \\
\hline Tontura/vertigem & 126 & 43,7 & $<0,0001$ & $2,69(1,74-4,16)$ \\
\hline Insônia & 175 & 48,5 & $<0,0001$ & $4,07(2,88-5,77)$ \\
\hline Problema urinário & 47 & 51,0 & $<0,0001$ & $2,79(1,89-4,14)$ \\
\hline Número de problemas de saúde & & & $<0,0001$ & \\
\hline 2 ou menos & 294 & 11,7 & & 1 \\
\hline 3 & 268 & 13,1 & & $1,08(0,56-2,08)$ \\
\hline 4 & 170 & 27,2 & & $2,22(1,46-3,38)$ \\
\hline 5 ou mais & 116 & 36,9 & & $2,86(1,63-5,01)$ \\
\hline Acidentes (último ano) & 56 & 23,7 & 0,2902 & $1,25(0,78-1,99)$ \\
\hline Violência (último ano) & 41 & 47,2 & 0,0002 & $2,82(1,87-4,26)$ \\
\hline IMC & & & 0,0165 & \\
\hline Eutrófico & 403 & 15,0 & & 1 \\
\hline Baixo peso & 47 & 10,4 & & $0,70(0,28-1,78)$ \\
\hline Sobrepeso & 245 & 21,1 & & $1,29(0,84-1,98)$ \\
\hline Obesidade & 153 & 28,0 & & $1,67(1,02-2,70)$ \\
\hline Autoavaliação da saúde & & & $<0,0001$ & \\
\hline Excelente & 129 & 6,7 & & 1 \\
\hline Muito boa & 176 & 6,8 & & $0,98(0,35-2,72)$ \\
\hline Boa & 476 & 19,7 & & $2,80(1,26-6,20)$ \\
\hline Ruim/muito ruim & 67 & 74,6 & & $10,16(4,43-23,28)$ \\
\hline
\end{tabular}

${ }^{*}$ Número de indivíduos na amostra não ponderada; ${ }^{* *}$ Teste qui-quadrado: valor de p; ${ }^{a}$ Modelo de regressão múltipla de Poisson. Variável de ajuste: idade. IMC: índice de massa corporal.

educação se destaque na associação com a saúde mental diante dos outros indicadores socioeconômicos? Segundo estudos, o acesso à educação gera habilidades cognitivas, assertividade e capacitação para tomar decisões, que contribuem com a independência, o controle da fertilidade, a qualidade da alimentação e o bem-estar econômico, fatores que influenciam na saúde física e mental ${ }^{20,21}$. Para alguns autores, a hipótese mais plausível é de que os recursos financeiros individuais proporcionados pelo trabalho remunerado sejam fundamentais na associação entre educação e saúde ${ }^{21}$. Ainda que o efeito independente de cada determinante social seja controverso, este estudo não encontrou associação entre TMC e renda após o ajuste pelas demais variáveis socioeconômicas e demográficas. Uma justificativa para esse resultado seria que a variável utilizada 
Tabela 4. Modelo hierárquico de regressão de Poisson de mulheres com 18 a 64 anos de Campinas, São Paulo. ISACamp 2008/2009.

\begin{tabular}{|c|c|c|c|c|c|c|}
\hline \multirow[b]{2}{*}{ Variáveis } & \multicolumn{2}{|c|}{ Primeira Etapa } & \multicolumn{2}{|c|}{ Segunda Etapa } & \multicolumn{2}{|c|}{ Terceira Etapa } \\
\hline & RP (IC 95\%) & $\begin{array}{l}\text { Valor } \\
\text { de p }\end{array}$ & RP (IC 95\%) & $\begin{array}{l}\text { Valor } \\
\text { de p }\end{array}$ & RP (IC 95\%) & $\begin{array}{l}\text { Valor } \\
\text { de p }\end{array}$ \\
\hline \multicolumn{7}{|l|}{ Faixa etária (anos) } \\
\hline 18 a 29 & 1 & & 1 & & 1 & \\
\hline 30 a 49 & $1,76(1,07-2,91)$ & 0,028 & $1,62(1,02-2,57)$ & 0,042 & $1,40(0,84-2,34)$ & 0,194 \\
\hline 50 a 64 & $1,66(0,97-2,82)$ & 0,061 & $1,45(0,85-2,47)$ & 0,169 & $1,05(0,53-2,08)$ & 0,884 \\
\hline \multicolumn{7}{|l|}{ Escolaridade (anos) } \\
\hline 13 ou mais & 1 & & 1 & & 1 & \\
\hline 9 a 12 & $2,55(1,54-4,20)$ & $<0,001$ & $2,72(1,66-4,47)$ & $<0,001$ & $2,50(1,60-3,92)$ & $<0,001$ \\
\hline 0 a 8 & $2,67(1,50-4,74)$ & 0,001 & $2,95(1,66-5,23)$ & $<0,001$ & $2,15(1,32-3,52)$ & 0,003 \\
\hline \multicolumn{7}{|l|}{ Ocupação } \\
\hline Trabalhadoras remuneradas & 1 & & 1 & & 1 & \\
\hline Desempregadas & $1,35(0,60-3,02)$ & 0,462 & $1,59(0,72-3,48)$ & 0,241 & $1,90(0,84-4,31)$ & 0,120 \\
\hline Aposentadas & $1,77(0,95-3,28)$ & 0,071 & $2,06(1,01-4,20)$ & 0,048 & $1,75(0,83-3,68)$ & 0,135 \\
\hline Donas de casa & $1,67(1,09-2,56)$ & 0,018 & $1,61(1,05-2,47)$ & 0,029 & $1,83(1,24-2,71)$ & 0,003 \\
\hline Estudantes & $1,86(0,56-6,22)$ & 0,306 & $2,29(0,76-6,85)$ & 0,137 & $2,56(0,87-7,53)$ & 0,087 \\
\hline \multicolumn{7}{|l|}{ Estado conjugal } \\
\hline Casada & 1 & & 1 & & 1 & \\
\hline Vive junto/amasiada & $1,16(0,72-1,88)$ & 0,535 & $1,10(0,70-1,73)$ & 0,658 & $1,09(0,70-1,71)$ & 0,686 \\
\hline Separada/divorciada/viúva & $1,67(1,07-2,60)$ & 0,025 & $1,61(1,05-2,48)$ & 0,029 & $1,23(0,84-1,84)$ & 0,280 \\
\hline Solteira & $1,29(0,75-2,20)$ & 0,349 & $1,11(0,68-1,83)$ & 0,658 & $1,11(0,72-1,72)$ & 0,615 \\
\hline \multicolumn{7}{|l|}{ Consumo fruta/verdura/legumes } \\
\hline Diário & & & 1 & & 1 & \\
\hline Não diário & & & $1,95(1,12-3,37)$ & 0,019 & $1,81(1,07-3,06)$ & 0,028 \\
\hline \multicolumn{7}{|l|}{ Duração diária do sono (horas) } \\
\hline$\geq 7$ & & & 1 & & 1 & \\
\hline$\leq 6$ & & & $2,66(1,87-3,78)$ & $<0,001$ & $2,05(1,51-2,80)$ & $<0,001$ \\
\hline \multicolumn{7}{|l|}{ Número de doenças crônicas } \\
\hline Nenhuma & & & & & 1 & \\
\hline 1 & & & & & $2,22(1,47-3,35)$ & 0,001 \\
\hline 2 & & & & & $2,35(1,36-4,07)$ & 0,003 \\
\hline 3 & & & & & $2,48(1,61-3,84)$ & $<0,001$ \\
\hline 4 ou mais & & & & & $3,86(2,28-6,52)$ & $<0,001$ \\
\hline \multicolumn{7}{|l|}{ Número de problemas de saúde } \\
\hline 2 ou menos & & & & & 1 & \\
\hline 3 & & & & & $0,90(0,49-1,69)$ & 0,760 \\
\hline 4 & & & & & $1,59(1,04-2,44)$ & 0,032 \\
\hline 5 ou mais & & & & & $1,94(1,21-3,12)$ & 0,007 \\
\hline \multicolumn{7}{|l|}{ Violência (último ano) } \\
\hline Não & & & & & 1 & \\
\hline $\operatorname{Sim}$ & & & & & $2,28(1,52-3,43)$ & $<0,001$ \\
\hline
\end{tabular}

foi a renda familiar per capita, uma vez que parte significativa das mulheres não possui renda, por não trabalhar fora de casa.

Congruente às argumentações apresentadas, é o fato de que juntamente com a educação, a ocupação foi o outro determinante social de grande importância na saúde mental das mulheres após os ajustes ${ }^{9,22,23}$. As donas de casa apresentaram 67\% mais TMC do que as trabalhadoras remuneradas. Juntos, o aumento da escolaridade feminina e a participação da mulher no mercado de trabalho fizeram com que as mulheres passas- 
sem a ser menos dependentes financeiramente de seus cônjuges. Mas, além do aspecto financeiro proporcionado pelo trabalho remunerado, a ocupação, como a escolaridade, também pode possibilitar às mulheres maior autonomia e poder de decisão, que coopera com a autoestima e confiança, e consequentemente melhora a satisfação com a vida ${ }^{24}$. A ocupação se destaca de forma particular por proporcionar uma rede social de apoio no ambiente de trabalho que facilita o autocontrole em situações de tensão e estresse ${ }^{25}$. Os resultados deste estudo revelam que as donas de casa apresentam mais prejuízos na saúde mental do que as trabalhadoras remuneradas, o que leva a crer que algumas características atreladas ao trabalho doméstico, bem como os estereótipos, podem ser prejudiciais aos aspectos mentais e emocionais. Autores consideram que a rotina, a monotonia do dia a dia de uma dona de casa, e a desvalorização do trabalho doméstico são fatores singulares e impactantes no prejuízo da saúde mental ${ }^{24-27}$.

A associação entre estado conjugal e saúde mental é amplamente pesquisada ${ }^{28,29}$. Mulheres separadas, divorciadas ou viúvas apresentaram prevalência de transtornos mentais $67 \%$ mais elevada do que mulheres casadas, resultado apoiado por pesquisa longitudinal, realizado no Canadá. Segundo esse estudo, tanto homens quanto mulheres que se separaram ou ficaram viúvos apresentaram níveis significativamente mais elevados de estresse do que aqueles que continuaram casados no período de dois anos ${ }^{30}$. Segundo uma meta-análise, com 126 artigos publicados nos últimos 50 anos, é a qualidade da relação conjugal que está relacionada aos indicadores de saúde. $\mathrm{O}$ relato de satisfação com o casamento foi associado a melhores marcadores biológicos como menor pressão arterial, risco de doenças cardiovascular e até mesmo mortalidade entre adultos ${ }^{28}$. Para os autores, a satisfação com o casamento é consequência do apoio social. O apoio social pode atenuar os efeitos estressantes do dia a dia. No casamento, indivíduos compartilham uma ampla variedade de atividades, que incluem refeições, tarefas domésticas, cuidado com os filhos, lazer, descanso, e recursos financeiros, muitas vezes, em maior grau do que aqueles que vivem juntos, mas não são casados. A ausência de apoio por parte do cônjuge pode ser motivo de conflito, insatisfação com o casamento, angústia psicológica e comprometimento da saúde física.

Em relação aos comportamentos relacionados à saúde, o papel da nutrição nos transtornos mentais tem tido um reconhecimento crescente por parte da literatura científica ${ }^{31,32}$. Este estudo constatou prevalência $93 \%$ mais elevada de TMC nas mulheres que não consumiam frutas, verduras ou legumes diariamente comparados às mulheres que faziam consumo diário desses alimentos. Resultado respaldado por outros estudos transversais internacionais, que verificaram associação entre a baixa ingestão ou deficiência de zinco, magnésio e ácido fólico (encontrados em níveis elevados nas frutas, legumes e verduras) e depressão e distimia em mulheres, após o ajuste por variáveis socioeconômicas e comportamentos ${ }^{33,34}$. Contudo, não podemos deixar de argumentar que uma dieta desequilibrada também pode refletir o comprometimento do autocuidado, atitude mais prevalente nas mulheres com transtornos mentais. Mas, segundo uma revisão sistemática sobre suplementação de ferro e zinco e sintomas depressivos de mulheres pré-menopausicas, de dez ensaios clínicos randomizados, sete estudos mostraram melhora no humor, memória e cognição das mulheres após a suplementação de ferro e de zinco ${ }^{35}$, indicando que a ausência desses minerais pode prejudicar a saúde mental.

Outro comportamento associado ao TMC entre as mulheres foi a duração do sono. É sabido que problemas relacionados com o sono estão entre os sinais que podem caracterizar algum transtorno mental a priori ${ }^{36}$. Como esperado, as mulheres que referiram dormir seis ou menos horas diárias apresentaram prevalência de TMC 2,66 vezes mais elevada do que aquelas que referiram dormir sete ou mais horas. Estudo realizado com idosos, também com os dados do ISACamp 2008/2009, apontou resultado similar, Lima et al. ${ }^{37}$ verificaram que as mulheres que dormiam cinco horas ou menos por dia apresentaram pior saúde mental, com os aspectos emocionais e a vitalidade prejudicados em relação às mulheres que dormiam de 7 a 8 horas. Há vários relatos na literatura de que o padrão de sono curto está associado aos prejuízos na saúde mental, como depressão, ansiedade, alteração de humor, tensão e fadiga ${ }^{38,39}$. De acordo com Faubel et al..$^{40}$, as mulheres que tinham um padrão de sono curto apresentaram pior qualidade de vida relacionada à saúde, sobretudo quanto aos aspectos emocionais, demonstrando a associação do estado de saúde e a funcionalidade do organismo, o bem estar físico e mental e o desempenho de atividades sociais.

É comum os transtornos mentais estarem acompanhados pelas doenças somáticas ${ }^{11,19,31}$, inclusive, a gravidade dos transtornos mentais 
pode estar relacionada com as comorbidades associadas. Mulheres que referiam quatro ou mais doenças crônicas apresentaram 3,75 vezes mais prevalência de TMC do que aquelas que referiram não ter doença. Com exceção ao diabetes e à alergia, este estudo identificou associação entre TMC e todas as morbidades e problemas de saúde pesquisados. Resultados do World Mental Health, pesquisa mundial com adultos de dezessete países, realizado pela OMS, revelam associação entre transtornos mentais e diabetes, asma, hipertensão, artrite, úlcera, doença do coração, problemas nas costas, dor de cabeça crônica, obesidade e múltiplas dores ${ }^{41}$. Dentre as doenças crônicas pesquisas, os problemas circulatórios foram os que apresentaram maior relevância na prevalência de TMC, as mulheres acometidas tiveram 2,58 vezes mais transtornos mentais. Em um estudo com mulheres, antecedentes de doenças cardiovasculares prévias, os sintomas depressivos aumentaram significativamente os riscos de desenvolver alguma doença cardiovascular e de mortalidade ${ }^{42}$. Ao analisar dados de estudos apresentados em uma conferência multidisciplinar sobre o tema, pesquisadores apontaram que o estresse crônico, a depressão e a ansiedade podem aumentar as taxas, as complicações e a mortalidade por doenças cardiovasculares à medida que podem afetar negativamente o autocuidado e causar alterações persistentes nas atividades hormonais e a desregulação do eixo hipotálamo-supra-renal ${ }^{43}$. Todavia, apesar da evidência da causalidade entre transtornos mentais e morbidades, como vem sendo apontado por alguns estudos, há necessidade de investigações mais aprofundadas. Há estudo que revela que doenças crônicas podem provocar aumento dos transtornos mentais à medida que afetam a qualidade de vida, pois interferem nas relações sociais, hábitos e rotinas ${ }^{44}$.

Por fim, outro fator associado ao TMC foi a violência contra a mulher, que ganha destaque por ser um dos fatores de maior impacto ${ }^{10,45}$. Mulheres que sofreram algum tipo de violência no último ano que antecedeu a entrevista apresentaram 2,28 mais transtornos mentais comuns comparadas àquelas que não relataram ter sofrido. Segundo a Organização Mundial da Saúde, a violência é a principal causa associada à depressão em mulheres ${ }^{46}$. Estudo realizado com $468 \mathrm{mu}-$ lheres vítimas de violência sexual na Região Metropolitana de Campinas apontou sintomas depressivos, ansiosos e alterações no sono em mais de metade das mulheres avaliadas ${ }^{47}$. Em pesquisa multicêntrica, em 10 países, incluindo o Brasil, os autores verificaram associação positiva entre violência e TMC, avaliado pelo SRQ-20, além da associação entre violência e pensamentos suicidas e tentativas de suicídios ${ }^{48}$. Segundo Kumar et al. ${ }^{10}$, a violência contra a mulher é um fenômeno extremamente complexo, que representa uma ameaça grave à saúde mental das mulheres, e está profundamente enraizado nas relações de gênero baseadas no poder, que envolvem questões de sexualidade, autoidentidade e também instituições sociais.

Algumas das limitações do estudo decorrem do corte transversal da pesquisa que não permite fazer inferências causais, e também do número total de entrevistadas que, apesar de adequado para estimar a maior parte das prevalências, mostra-se insuficiente para algumas categorias menos frequentes de algumas variáveis. Quanto ao instrumento utilizado, o SRQ-20, foi submetido a estudo de validação na população urbana brasileira ${ }^{49}$ e se destaca por ser amplamente utilizado em inquéritos de base populacional devido a sua simples aplicação, que pode ser por meio do autopreenchimento ou de entrevista realizada por entrevistadores leigos e treinados, como foi o caso desta pesquisa. Entretanto, ressalta-se que o SRQ-20 não estabelece categorias diagnósticas como na Classificação Internacional de Doenças - 10 (CID-10) e no Diagnostic and Statistical Manual - V (DSM-V), sendo, portanto, um instrumento de rastreamento de casos suspeitos de transtornos mentais comuns.

Este estudo destaca que mulheres mais velhas, com baixa escolaridade, donas de casa, separadas ou viúvas, com dieta alimentar inadequada, que dormem pouco, doentes e que sofrem violência fazem parte dos segmentos mais vulneráveis ao transtorno mental comum e, portanto, devem ser tratadas com prioridade pelos serviços de saúde. É necessária uma sensibilização na avaliação realizada pelo profissional de saúde, com atenção às condições socioeconômicas da mulher, e compreensão dos fatores de risco específicos aos problemas mentais na história de vida de cada uma, como o estilo de vida, a presença prévia de morbidades e a história de violência. Dada a associação entre TMC e doenças crônicas, problemas de saúde e violência, e as consequências clínicas das comorbidades, que agravam ainda mais os transtornos mentais, esses fatores de risco devem ser objetos de pesquisas, e trabalhados na prevenção de TMC e no auxílio para o diagnóstico na clínica médica. Diagnosticar precocemente mulheres com TMC, bem como acompanhá-las e tratá-las, pode reduzir os impactos dos transtornos men- 
tais na qualidade de vida, minimizando o sofrimento e os resultados adversos na saúde delas e de suas famílias, e os custos aos serviços de saúde.

\section{Colaboradores}

C Senicato trabalhou na concepção do artigo, realizou as análises estatísticas e a redação do texto. RCS Azevedo participou da revisão do artigo. MBA Barros participou do delineamento e concepção da pesquisa e orientou a concepção, as análises estatísticas e a redação do artigo.

\section{Agradecimentos}

À Fundação de Amparo à Pesquisa do Estado de São Paulo (FAPESP), pela bolsa de doutorado de CS. Ao Conselho Nacional de Desenvolvimento Científico e Tecnológico (CNPq) pelo financiamento da pesquisa e pela bolsa de produtividade de MBAB; ao Ministério da Saúde e à Secretaria de Saúde de Campinas pelo apoio financeiro para a realização do inquérito (Convênio Unicamp/ Funcamp/SMS).

\section{Referências}

1. World Health Organization (WHO). The World Health Report 2001: mental health new understanding, new hope. Geneva: WHO; 2001.

2. Goldberg D, Huxley P. Commom mental disorders: a bio-social model. London: Tavistock; 1992.

3. Steel Z, Marnane C, Iranpour C, Chey T, Jackson JW, Patel, V, Silove D. The global prevalence of common mental disorders: a systematic review and meta-analysis 1980-2013. Int J Epidemiol 2014; 43(2):476-493.

4. Mokdad AH, Forouzanfar MH, Daoud F, Mokdad AA, El Bcheraoui C, Moradi-Lakeh M, Kyu HH, Barber RM, Wagner J, Cercy K, Kravitz H, Coggeshall M, Chew A, O'Rourke KF, Steiner C, Tuffaha M, Charara R, AlGhamdi EA, Adi Y, Afifi RA, Alahmadi H, Al Buhairan F, Allen N, Al Mazroa M, Al-Nehmi AA, Al Rayess Z, Arora M, Azzopardi P11, Barroso C, Basulaiman M, Bhutta ZA, Bonell C, Breinbauer C, Degenhardt L, Denno D, Fang J, Fatusi A, Feigl AB, Kakuma R, Karam N, Kennedy E, Khoja TA, Maalouf F, Obermeyer CM, Mattoo A, McGovern T, Memish ZA, Mensah GA, Patel V, Petroni S, Reavley N, Zertuche DR, Saeedi M, Santelli J, Sawyer SM, Ssewamala F, Taiwo K, Tantawy M, Viner RM, Waldfogel J, Zuñiga MP, Naghavi M, Wang H, Vos T, Lopez AD, Al Rabeeah AA, Patton GC, Murray CJ. Global burden of diseases, injurie, and risk factors for Young people's health during 1990-2013: a systematic analysis for the Global Burden of Disease Study 2013. Lancet 2016; 387(10037):2383-2401.

5. World Health Organization (WHO), UNFPA. Mental health aspects of women's reproductive health: a review of the literature. Geneva: WHO Press; 2009.

6. Lima MCP, Menezes PR, Carandina L, Cesar CLG, Barros MBA, Goldbaum M. Transtornos mentais comuns e uso de psicofármacos: impacto das condições socioeconômicas. Rev Saude Publica 2008; 42(4):717-723.

7. Simon GE. Social and economic burden of mood disorders. Biol Psychiatry 2003; 54(3):208-215.
8. Joel D, Berman Z, Tavor I, Wexler N, Gaber O, Stein Y, Shefi N, Pool J, Urchs S, Margulies DS, Liem F, Hänggi J, Jäncke L, Assaf Y. Sex beyond the genitalia: the human brain mosaic. PNAS 2015; 112(50):15468-15473.

9. Araújo TM, Pinho PS, Almeida MMG. Prevalência de transtornos mentais comuns em mulheres e sua relação com as características sociodemográficas e o trabalho doméstico. Rev Bras Saúde Matern Infant 2005; 5(3):337-348.

10. Kumar A, Nizamie SH, Srivastava NK. Violence against women and mental health. Mental Health Prevention 2013; 1:4-10.

11. Boing AF, Melo AR, Boing AC, Moretti-Pires RO, Peres KG, Peres MA. Associação entre depressão e doenças crônicas: estudo populacional. Rev Saude Publica 2012; 46(4):617-623.

12. Santos ÉG, Siqueira MM. Prevalência dos transtornos mentais na população adulta brasileira: uma revisão sistemática de 1997 a 2009. J Bras Psiquiatr 2010; 59(3):238-246.

13. Ludermir AB, Melo filho DA. Condições de vida e estrutura ocupacional associadas a transtornos mentais comuns. Rev Saude Publica 2002; 36(2):213-221.

14. Lima CT, Freire ACC, Silva APB, Teixeira RM, Farrel $\mathrm{M}$, Prince M. Concurrent and construct validity of the AUDIT in urban Brazilian sample. Alcohol 2005; 40(6):584-589.

15. Craig CL, Marshall AL, Sjostrom M, Bauman AE, Booth ML, Ainsworth BE, Pratt M, Ekelund U, Yngve A, Sallis JF, Oja P. International physical activity questionnaire: 12-country reliability and validity. Med Sci Sports Exerc 2003; 35(8):1381-1395.

16. World Health Organization (WHO). Global recommendations on physical activity for health. Genebra: WHO; 2010. [cited 2015 Mar 5]. Available from: http://whqlibdoc.who.int/publications/2010/9789241599979_ eng.pdf 
17. Brasil. Ministério da Saúde (MS). Guia alimentar para a população brasileira: promovendo a alimentação saudável. Brasília: MS; 2008. [acessado 2015 Mar 04]. Disponível em: http://bvsms.saude.gov.br/bvs/publicacoes/guia_alimentar_populacao_brasileira_2008.pdf

18. Senicato C, Barros MBA. Social inequality in health among women in Campinas, São Paulo State, Brazil. Cad Saude Publica 2012; 28(10):1903-1914.

19. Coelho FMC, Pinheiro RT, Horta BL, Magalhães PVS, Garcias CMM, Silva CV. Common mental disorders and chronic non-communicable diseases in adults: a population-based study. Cad Saude Publica 2009; 25(1):59-67.

20. Stewart DE, Ashraf IJ, Munce SE. Women's mental health: a silent cause of mortality and morbidity. Int $J$ Gynaecol Obstet 2006; 94(3):343-349.

21. Bates LM, Berkman LF, Glymour M. Socioeconomic determinants of women's health: the changing landscape of education, work, and marriage. Women Health 2013; 2:671-683.

22. Noordt M, Ijzelenberg H, Droomers M, Proper KI. Health effects of employment: a systematic review of prospective studies. Occup Environ Med 2014; 71(10):730-736.

23. Araújo TM, Almeida MMG, Santana CC, Araújo EM, Pinho PS. Transtornos mentais comuns em mulheres: estudo comparativo entre dona-de-casa e trabalhadoras. $R$ Enferm UERJ 2006; 14(2):260-269.

24. Brown GW, Harris TO. Social origins of depression: a study of psychiatric disorder in women. London: Tavistock; 1978.

25. Klumb PL, Lampert T. Women, work, and well-being 1950-2000: a review and methodological critique. Soc Sci Med 2004; 58(6):1007-1024.

26. Lennon MC. Women, work, andwell-being: the importance of work conditions. J Health Soc Behav 1994; 35(3):235-247.

27. Sorensen G, Verbrugge LM. Women, work, and health. Ann Rev Public Health 1987; 8:235-251.

28. Robles TF, Slatcher RB, Trombello JM, McGinn MM. Marital quality and health: a meta-analytic review. Psychol Bull 2014; 140(1):140-187.

29. Lindstrom M, Rosvall M. Marital status, social capital, economic stress, and mental health: a population-based study. The Social Science Journal 2012; 49(3):339-342.

30. Strohschein L, McDonough P, Monette G, Shao Q. Marital transitions and mental health: are there gender differences in the short-term effects of marital status change? Soc Sci Med 2005; 61(11):2293-2303.

31. Zender R, Olshansky E. Women's mental health: depression and anxiety. Nurs Clin N Am 2009; 44(3):355-364.

32. Murphy PK, Wagner CL. Vitamin D and mood disorders among women: na integrative review. J Midwifery Womens Health 2008; 53(5):440-446.

33. Jacka FN, Maes M, Pasco JA, Williams LJ, Berk M. Nutrient intakes and the common mental disorders in women. J Affect Disord 2012; 141(1):79-85.

34. Jacka FN, Pasco JA, A Mykletun, Williams LJ, Berk M. Diet as a modifiable risk factor for the common mental disorders: evidence and mechanisms. J Affect Disord 2010; 122(Supl. 1):17-18.

35. Lomagno KA, Hu F, Riddell LJ, Booth AO, Szymlek Gay EA, Nowson CA, Byrne LK. Increasing iron and zinc in pre-menopausal women and its effects on mood and cognition: a systematic review. Nutrients 2014; 6(11):5117-5141.
36. Soares CN. Depression during the menopausal transition: window of vulnerability or continuum of risk? Menopause 2008; 15(2):207-209.

37. Lima MG, Barros MBA, Alves MCGP. Sleep duration and health status sef-assessment (SF-36) in the elderly: a population-based study (ISA-Camp 2008). Cad Saude Publica 2012; 28(9):1674-1684.

38. Krueger PM, Friedman EM. Sleep duration in the United States: a cross-sectional population-based study. Am J Epidemiol 2009; 169(9):1052-1063.

39. Birchler-Pedross A, Schröder CM, Münch M, Knoblauch V, Blatter K, Schnitzler-Sack C, Wirz-Justice A, Cajochen C. Subjective well-being is modulated by cicardianphase, sleep pressure, age and gender. J Biol Rhythm 2009; 24(3):232-242.

40. Faubel R, Lopez-Garcia E, Guallar-Castillón P, BalboaCastillo T, Gutiérrez-Fisac JL, Banegas JR, Rodríguez -Artalejo F. Sleep duration and health-related quality of life among older adults: a population-based cohort in Spain. Sleep 2009; 32(8):1059-1068.

41. Scott KM, Bruffaerts R, Tsang A, Ormel J, Alonso J, Angermeyer MC, Benjet C, Bromet E, de Girolamo G, de Graaf R, Gasquet I, Gureje O, Haro JM, He Y, Kessler RC, Levinson D, Mneimneh ZN, Oakley Browne MA, Posada-Villa J, Stein DJ, Takeshima T, Von Korff M. Depression-anxiety relationships with chronic physical conditions: Results from the World Mental Health surveys. J Affect Disord 2007; 103(1-3):113-120.

42. Whang W, Kubzansky LD, Kawachi I, Rexrode KM, Kroenke CH, Glynn RJ, Garan H, Albert CM. Depression and risk of sudden cardiac death and coronary heart disease in women. J Am Coll Cardiol 2009; 53(11):950-958.

43. Roy-Byrne R, Davidson KW, Kessler RC, Asmundson GJ, Goodwin RD, Kubzansky L, Lydiard RB, Massie MJ, Katon W, Laden SK, Stein MB. Anxiety disorders and comorbid medical illness. Gen Hosp Psychiatry 2008; 30(3):208-225.

44. Berber JSS, Kupek E, Berber SC. Prevalência de depressão e sua relação com qualidade de vida em pacientes com síndrome da fibromialgia. Rev Bras Reumatol 2005; 45(2):47-54.

45. Machado CL, Azevedo RCS, Facuri CO, Vieira MJN, Fernandes AMS. Post traumatic stress disorder, depression and hopelessness in women who are victims of sexual violence. Int J Gynaecol Obstet 2011; 113(Supl. 1):58-62.

46. World Health Organization (WHO). Women's mental health: an evidence based review. Geneva: WHO; 2000.

47. Facuri CO. Características sociodemográficas e sintomas psíquicos de mulheres vítimas de violência sexual [dissertação]. Campinas: Unicamp; 2012.

48. Ellsberg M, Jansen HAFM, Heise L, Watts CH, Garcia-Moreno C ; WHO Multi-country Study on Women's Health and Domestic Violence against Women Study Team. Intimate partner violence and women's physical and mental health in the WHO multi-country study on women's health and domestic violence: an observational study. Lancet 2008; 371(9619):1165-1172.

49. Mari JJ, Williams P. A validity study of a psychiatric screening questionnaire (SRQ-20) in primary care in the city of Sao Paulo. Br J Psychiatry 1986; 148:23-26.

Artigo apresentado em 19/05/2016

Aprovado em 29/09/2016

Versão final apresentada em 01/10/2016 